\title{
A multi-centre experience of ablation index for evaluating lesion delivery in cavotricuspid isthmus dependent atrial flutter
}

\author{
Edd Maclean ${ }^{1}$, Ron Simon ${ }^{2}$, Richard Ang ${ }^{3}$, Gurpreet Dhillon ${ }^{3}$, Syed Ahsan ${ }^{3}$, Fakhar Khan ${ }^{2}$, \\ Mark Earley ${ }^{2}$, Pier Lambiase ${ }^{3}$, James Rosengarten ${ }^{3}$, Anthony Chow ${ }^{2}$, Mehul Dhinoja ${ }^{4}$, Rui \\ Providencia $^{3}$, Vias Markides ${ }^{5}$, Tom Wong ${ }^{5}$, Ross Hunter ${ }^{2}$, and Jonathan Behar ${ }^{5}$ \\ ${ }^{1}$ Queen Mary University of London \\ ${ }^{2}$ Barts Health NHS Trust \\ ${ }^{3}$ Saint Bartholomew's Hospital \\ ${ }^{4}$ Barts and The London NHS Trust \\ ${ }^{5}$ Royal Brompton and Harefield NHS Foundation Trust
}

July 6,2020

\begin{abstract}
Introduction Anatomical studies demonstrate significant variation in cavotricuspid isthmus (CTI) architecture. We hypothesised that ablation index (AI) may further our understanding of energy delivery across the CTI. Methods 38 patients underwent CTI ablation at two Cardiothoracic hospitals. Operators delivered 682 lesions in total with a target AI of 600Wgs. Ablation parameters were recorded every 10-20ms. Post hoc, Visitags were trisected according to CTI position: inferior vena cava (IVC), middle (Mid), or ventricular (V) lesions. Results There were no complications. 97.4\% of patients $(\mathrm{n}=37)$ remained in sinus rhythm at $6.6 \pm 3.3$ months' follow-up. For the whole CTI, peak AI correlated with mean impedance drop (ID) (R2=0.89, p $<0.0001)$. However, analysis by anatomical site demonstrated a non-linear relationship Mid CTI $(\mathrm{R} 2=0.15, \mathrm{p}=0.21)$. Accordingly, whilst mean AI was highest Mid CTI (IVC: $473.1 \pm 122.1 \mathrm{Wgs}$, Mid: $539.6 \pm 103.5 \mathrm{Wgs}$, V: $486.2 \pm 111.8$ Wgs, ANOVA p<0.0001), mean ID was lower (IVC: $10.7 \pm 7.5$, Mid: $9.0+-6.5, \mathrm{~V}: 10.9+-7.3, \mathrm{p}=0.011$ ), and rate of ID was slower (IVC: $0.37+-0.05 / \mathrm{s}, \mathrm{Mid}$ : $0.18+-0.08 / \mathrm{s}, \mathrm{V}: 0.29+-0.06 / \mathrm{s}, \mathrm{p}<0.0001)$. Mean contact force was similar at all sites, however temporal fluctuations in contact force (IVC: $19.3+-12.0 \mathrm{mg} / \mathrm{s}$, Mid: $188.8+-92.1 \mathrm{mg} / \mathrm{s}, \mathrm{V}: 102.8+-32.3 \mathrm{mg} / \mathrm{s}, \mathrm{p}<0.0001$ ) and catheter angle (IVC: $0.42 \mathrm{deg} / \mathrm{s}, \mathrm{Mid}$ : $3.4 \mathrm{deg} / \mathrm{s}, \mathrm{V}: 0.28 \mathrm{deg} / \mathrm{s}, \mathrm{p}<0.0001)$ were greatest Mid CTI. Use of a long sheath attenuated these fluctuations and improved ablation efficacy. Conclusions Ablation characteristics vary across the CTI. At the Mid CTI, operators should appreciate that higher AI values do not necessarily deliver more effective ablation; this may be explained by localised fluctuations in catheter angle and contact force.
\end{abstract}

\section{Keywords}

Ablation index

Atrial flutter

Catheter ablation

Force sensing

Cavo-tricuspid isthmus

Funding 
This research did not receive any specific grant from agencies in the public, commercial, or not-for-profit sectors

Abbreviations

AI - Ablation Index

$\mathrm{AF}$ - atrial fibrillation

CTI - cavotricuspid isthmus

ID - impedance drop

- Ohms

PVI - pulmonary vein isolation

LVEF - left ventricular ejection fraction

Wgs - Watts/grams/second

Introduction

The advent of contact force-sensing catheters has delivered important data on ablation lesion size, safety and efficacy, and their application is well-described during pulmonary vein isolation (PVI) for atrial fibrillation $(\mathrm{AF})$. Using a weighted formula, catheter contact force $(\mathrm{g})$ can be combined with the duration (s) and power (W) of a radiofrequency application to calculate Ablation Index (AI). Expressed as a continuous value in Wgs, AI has been shown to predict lesion diameter and depth during AF ablation, and left atrial procedures guided by site-specific AI targets have demonstrated more enduring PVI and a comparable safety profile versus conventional ablation techniques. AI infers the energy delivered by the ablation catheter; this differs from impedance drop (ID) which describes local impedance changes at the blood-tissue interface and infers tissue receipt of injury. Whilst AI is known to correlate with ID, the strength of this relationship attenuates with procedural variables such as catheter angle of incidence and irrigation techniques; as such, there may be important limitations when relying on AI alone to guide lesion delivery .

Radiofrequency ablation of cavo-tricuspid isthmus (CTI) dependent ('typical') atrial flutter achieves acute success (i.e. bidirectional block) in over $90 \%$ of cases, and carries a class IA recommendation as a treatment strategy in the ESC's 2019 Supraventricular Tachycardia (SVT) guidelines . Despite this, a significant proportion of CTI ablations can prove technically challenging, and hence novel predictors of acute and long-term efficacy remain desirable. Autopsy studies have demonstrated marked heterogeneity in CTI architecture; Klimek-Piotrowska et al. (2016) dissected 140 human hearts and found that, when compared to the anterior or posterior margins, the middle CTI frequently harbours distinct morphological variations such as trabeculae $(62.1 \%)$ or recesses $(25 \%)$. Peri-ablation imaging data has also shown that structural anomalies - such as the presence of pouches, angular crypts or tricuspid regurgitation - result in prolonged procedure times and poorer outcomes . Accordingly, data from contact-force sensing catheters suggests that site-specific inconsistencies in isthmus tissue contact may be responsible for procedural failure. The prevalence of complex CTI anatomy has led some authors to suggest that pre-procedural imaging, such as cardiac MRI or right atrial angiography, would promote more patient-specific ablation strategies and hence improve outcomes .

\section{Hypothesis}

We hypothesised that AI may provide important insights into lesion delivery across the CTI during ablation of typical atrial flutter. Using established 3D electro-anatomical mapping systems and ablation catheters, examining local variations in ablation lesion characteristics may elucidate the mechanisms which impede enduring bidirectional block or contribute to complications. An appreciation of these relationships may promote a more prescriptive approach to CTI ablation without the use of additional resources such as perior intraprocedural imaging techniques.

Methods 


\section{Ethics}

This project was registered with the local clinical effectiveness unit. Consenting patients underwent procedures which were clinically indicated, without randomisation or allocation, and which made use well-established mapping and ablation techniques. As such, the work was consistent with Clinical Service Development in line with the UK's Health Research Authority recommendations, and no specific additional ethical approval was required.

Procedure

Patients with no previous CTI ablation underwent radiofrequency ablation of CTI-dependent atrial flutter at two tertiary Cardiothoracic hospitals in the UK from 2019-2020. Procedures were performed on uninterrupted oral anticoagulation, either under general anaesthetic or with conscious intravenous sedation. Ultrasound-guided femoral venepuncture was performed following local anaesthetic administration, and a quadripolar or decapolar catheter was positioned in the coronary sinus. A contact-force sensing ablation catheter (Thermocool SmartTouch ${ }^{\mathrm{TM}}$, Biosense Webster, Diamond Bar, California) was passed to the right atrium. Electro-anatomical mapping was performed using CARTO software (v3, Biosense Webster). For patients presenting in atrial flutter, entrainment discerned CTI dependence, whereas patients in sinus rhythm underwent empirical CTI ablation during pacing of the proximal coronary sinus at $600 \mathrm{~ms}$. Point-by-point ablation was delivered from the ventricular margin of the CTI progressing towards the inferior vena cava, maintaining a 6 o'clock alignment in the left anterior oblique projection as per standard clinical protocol. The use of additional sheaths to guide ablation was at the operators' discretion. Ablation VisiTag ${ }^{\text {TM }}$ settings were pre-specified to accept $5 \mathrm{~mm}$ of catheter drift, and force-over-time (FOT) constraints of $5 \mathrm{~s}, 25 \%$ in conjunction with $3 \mathrm{~g}$ minimum force. Operators were advised to deliver ablation lesions with a peak AI of $600 \mathrm{Wgs}$ at $45-50 \mathrm{~W}$. These recommendations were based on a retrospective analysis performed at our institution which demonstrated safe and effective CTI ablation with clusters of lesions in this range of AI (18). In the event of visual macro-displacement, locations were discarded. Saline irrigation flow rate was $2 \mathrm{ml} / \mathrm{min}$ during mapping and $17 \mathrm{ml} / \mathrm{min}$ during ablation. Impedance was measured between the catheter tip and a ground patch on the patient's right thigh. If bidirectional CTI block was achieved, this was reassessed after 15 minutes' observation and consolidative ablation lesions delivered as required. In the absence of complications, patients were discharged the same day if the procedure was performed under sedation, or the following day if performed under general anaesthetic.

Data extraction

Post hoc, VisiTags were anatomically trisected according to their position on the CTI: IVC end (IVC), middle CTI (Mid), or ventricular end (V). Lesion characteristics were subsequently extracted and aligned with time stamps to allow assessment of temporal changes, including contact force (every 50ms), impedance, impedance drop and ablation index (every 10-20ms), catheter angle in the axial and lateral planes (every 10-20ms), and power (every 100ms). It has been demonstrated that precipitous rises in impedance are associated with thrombus and steam formation, and a variety of methods for assessing change in impedance during catheter ablation have been published previously, including total impedance drop, overall median impedance drop, or median impedance drop after 10 seconds of ablation (2) (9) (19). During our data cleaning, very rare transient spikes and troughs (lasting $<50 \mathrm{~ms}$ ) in impedance - and consequently the running calculations of impedance drop - were noted. The examination of other contemporaneous lesion characteristics (e.g. contact force) suggested that these data points were real, and as their ramifications on ablation safety were potentially significant, they were included in further analyses. However, these outlying values rendered the measurement of total impedance drop less reliable, and so a mean ID was instead calculated from all the values recorded throughout the duration of each radiofrequency application. The mean ID was assigned as a surrogate marker of ablation efficacy. Total energy delivery was assessed in terms of peak ablation index; this was defined as the maximum recorded AI (Wgs) measured during each lesion; for all VisiTags, this corresponded to the final recorded value.

Follow-up 
Patients underwent clinical review with ECG analysis at 3 months post procedure, with a further review at 12 months or sooner if necessitated by symptoms. Anticoagulation was continued according to $\mathrm{CHA}_{2} \mathrm{DS}_{2}-\mathrm{VASc}$ score, and anti-arrhythmic drugs were adjusted according to patient preference and physicians' discretion.

Statistical analysis

Data were analysed in R (x64, v. 3.5.2.) and XLSTAT (Addinsoft, v. 2020.1). Categorical group parameters were compared using Chi square tests. The Shapiro-Wilk test identified whether or not data were normally distributed. Subsequently, continuous data were compared with two-tailed $\mathrm{T}$ tests or analysis of variance (ANOVA) with post-hoc Tukey HSD testing for normally distributed data, or with the Mann-Whitney U or Kruskal-Wallis test for non-normally distributed data. Correlation was assessed using Pearson's coefficient for normally distributed data or the Spearman rank correlation if non-normally distributed. Data are presented as mean $\pm \mathrm{SD}$ or median (interquartile range). The level of significance was set at $\mathrm{p}<0.05$.

Results

38 individuals were included in this study; clinical characteristics and baseline ablation data are shown in table 1.14 cases made use of additional long sheaths (11 Swartz SR0, Abbott/St Jude Medical; two Agilis ${ }^{\mathrm{TM}}$, Abbott/St Jude Medical, one Vizigo ${ }^{\mathrm{TM}}$, Biosense Webster) to assist ablation. Acute success (bidirectional block) was achieved in all 38 cases (100\%). There were no complications, and no steam pops were recorded. After a mean follow-up of $6.6 \pm 3.3$ months, $97.4 \%(\mathrm{n}=37)$ of patients were in sinus rhythm.

Table 1: clinical characteristics and baseline ablation data

\begin{tabular}{ll}
\hline Clinical characteristics & Clinical characteristics \\
\hline Age (years) & $71(15.5)$ \\
Male & $89.5 \%(\mathrm{n}=34)$ \\
Duration of atrial flutter (months) & $8.5(8)$ \\
Hypertension & $23.7 \%(\mathrm{n}=9)$ \\
Diabetes & $15.8 \%(\mathrm{n}=6)$ \\
Smoking history & $13.2 \%(\mathrm{n}=5)$ \\
Ischaemic heart disease (previous PCI or CABG) & $10.5 \%(\mathrm{n}=4)$ \\
Congenital heart disease & $2.6 \%(\mathrm{n}=1 ;$ coarctation of the aorta) \\
Beta blocker & $86.8 \%(\mathrm{n}=33)$ \\
Class III anti-arrhythmic drug & $15.8 \%(\mathrm{n}=6)$ \\
Anticoagulated with DOAC (remainder warfarin) & $92.1 \%(\mathrm{n}=35)$ \\
LV ejection fraction (\%) & $55(10)$ \\
Baseline ablation data & $\mathrm{Baseline}$ ablation data \\
General anaesthetic & $23.7 \%(\mathrm{n}=9)$ \\
Procedure started in sinus rhythm & $23.7 \%(\mathrm{n}=9)$ \\
Long sheath used & $36.8 \%(\mathrm{n}=14)$ \\
Procedure time (minutes) & $61.4 \pm 37.1$ \\
Fluoroscopy time (minutes) & $2.7 \pm 4.7$ \\
Dose area product (cGy/cm $\left.{ }^{2}\right)$ & $78.6 \pm 254$ \\
\hline
\end{tabular}

Parameters listed for 38 patients undergoing CTI ablation as \% (n), mean \pm SD, or median (IQR)

Ablation lesion characteristics are summarised in table 2.

Table 2: ablation lesion characteristics across the CTI 


\begin{tabular}{|c|c|c|c|c|c|}
\hline Parameter & Whole CTI & IVC & Middle & Ventricular & $\mathrm{p}$ value \\
\hline Peak AI (Wgs) & $499.6 \pm 91.6$ & $473.1 \pm 122.1$ & $539.6 \pm 103.5$ & $486.2 \pm 111.8$ & $\begin{array}{l}\text { Mid vs IVC or V } \\
\mathrm{p}<0.0001 I V C \\
\text { vs } V p=0.63\end{array}$ \\
\hline Power (W) & $45.1 \pm 4.3$ & $45.0 \pm 4.3$ & $44.8 \pm 4.6$ & $45.8 \pm 4.1$ & All $p>0.1$ \\
\hline $\begin{array}{l}\text { Lesion } \\
\text { duration (s) }\end{array}$ & $23.7 \pm 13.2$ & $21.9 \pm 14.5$ & $28.9 \pm 12.4$ & $22.0 \pm 15.2$ & $\begin{array}{l}\text { Mid vs IVC or V } \\
\mathrm{p}<0.0001 I V C \\
\text { vs } V p=0.98\end{array}$ \\
\hline $\begin{array}{l}\text { Contact } \\
\text { force }(g)\end{array}$ & $10.5 \pm 7.3$ & $10.4 \pm 7.2$ & $10.6 \pm 7.6$ & $10.3 \pm 7.3$ & All $p>0.1$ \\
\hline $\begin{array}{l}\text { Impedance } \\
\text { drop (Ohms) }\end{array}$ & $9.7 \pm 5.7$ & $10.7 \pm 7.5$ & $9.0 \pm 6.5$ & $10.9 \pm 7.3$ & $\begin{array}{l}\text { Mid vs IVC } \\
\mathrm{p}=0.011, \text { Mid Vs } \\
\mathrm{V} \mathrm{p}=0.005 I V C \\
\text { vs } V p=0.76\end{array}$ \\
\hline $\begin{array}{l}\text { Rate of } \\
\text { impedance } \\
\text { drop } \\
(\text { Ohms } / \mathrm{s})\end{array}$ & $0.28 \pm 0.03$ & $0.37 \pm 0.05$ & $0.18 \pm 0.08$ & $0.29 \pm 0.06$ & All $\mathrm{p}<0.0001$ \\
\hline $\begin{array}{l}\text { Temperature } \\
\left({ }^{\circ} \mathbf{C}\right)\end{array}$ & $37.4 \pm 4.6$ & $38.1 \pm 4.4$ & $36.2 \pm 5.4$ & $37.8 \pm 4.0$ & $\begin{array}{l}\text { Mid vs IVC or V } \\
\mathrm{p}<0.0001 I V C \\
\text { vs } V p=0.52\end{array}$ \\
\hline
\end{tabular}

Lesion components listed for the CTI as a whole and stratified according to anatomical site. Significant p values $(<0.05)$ are highlighted in bold.

Across all procedures, a total of 682 ablation lesions were delivered (IVC: 206, Mid CTI: 242, V: 234) and $6,187,716$ data points extracted. The number of ablation lesions per procedure ranged from 5 to 44 . For the whole CTI, there was a strong correlation between peak AI and mean impedance drop (figure $1-\mathrm{R}^{2}=0.89$, $\mathrm{p}<0.0001)$. However, analysis by anatomical site demonstrated significant variation in this relationship (figure 2). 


\section{Peak ablation index versus mean impedance drop (average of all anatomical sites)}

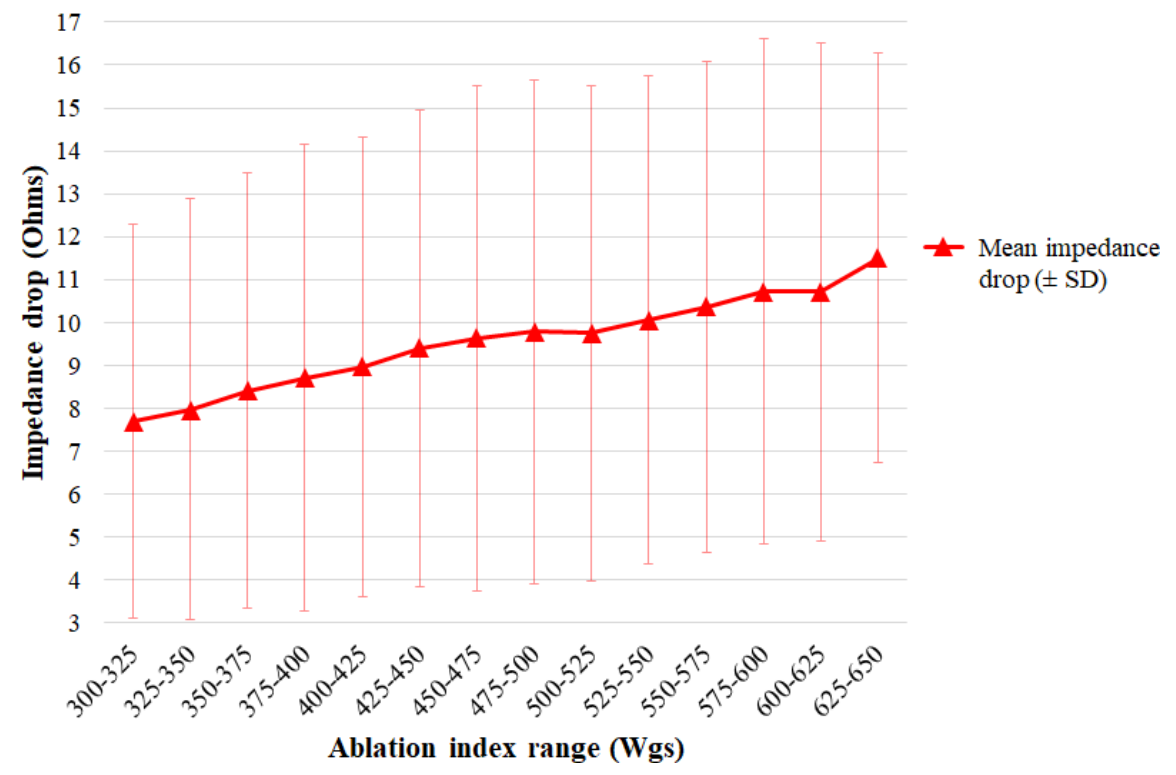

Figure 1: Relationship between peak ablation index (Wgs) and mean impedance drop (Ohms; mean $\pm \mathrm{SD}$ ) for all CTI lesions. Pearson's $\mathrm{R}^{2}=0.89, \mathrm{p}<0.0001$

\section{Peak ablation index versus mean impedance drop according to CTI anatomical site}

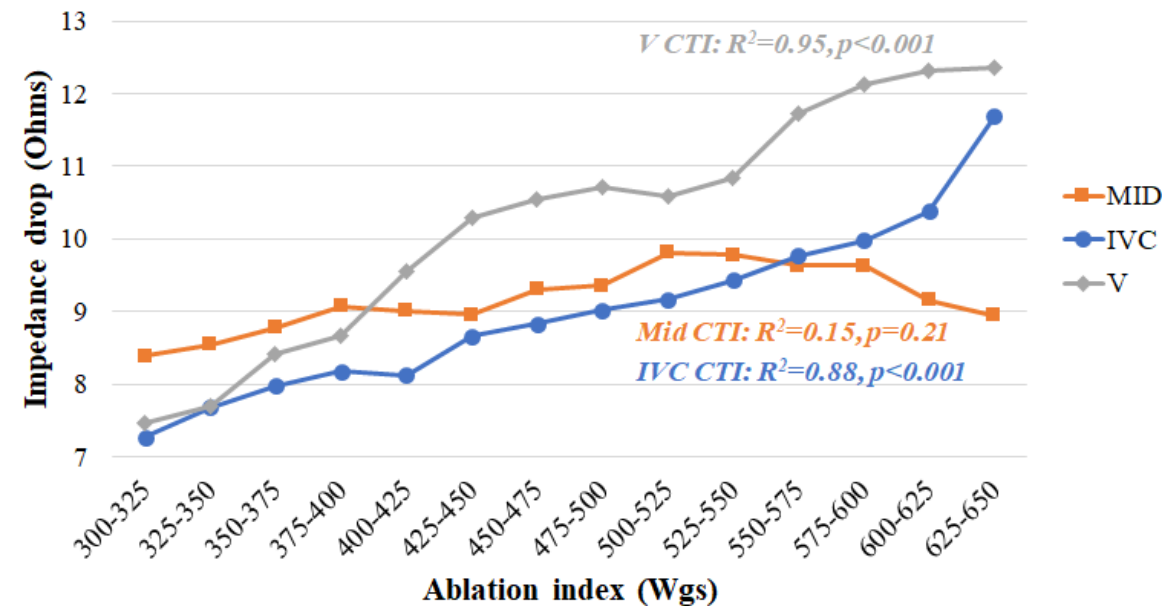

Figure 2: Relationship between peak ablation index (Wgs) and mean impedance drop (Ohms) per lesion according to CTI anatomical site $\left(\mathrm{V} \mathrm{CTI}^{2}=0.95, \mathrm{p}<0.0001\right.$, Mid CTI $\mathrm{R}^{2}=0.15, \mathrm{p}=0.21$, IVC CTI $\mathrm{R}^{2}=0.88$, $\mathrm{p}<0.0001)$

Whilst AI and impedance drop maintained a strong linear association at the V and IVC margins, at the Mid CTI linearity was less apparent with a qualitative plateau in impedance drop noted beyond AI of 500Wgs (Figure $2-\mathrm{R}^{2}=0.15, \mathrm{p}=0.21$ ). Longer lesion duration was also required Mid CTI to achieve the target AI (table 2). In addition, whilst mean peak AI was highest Mid CTI, mean impedance drop was lower, and rate 
of impedance drop was slower Mid CTI (table 2). Mean contact force and power were similar at all sites, however mean temperature was lowest Mid CTI (table 2).

Given that these findings suggested that comparable energy applications (according to AI) produced inconsistent degrees of tissue injury (by ID) at different CTI sites despite similar mean contact force and power, further analysis was performed to examine the catheter-tissue interface.

Temporal fluctuation in contact force was determined by examining the change in recorded catheter force (g) every 50ms. A mean value for each lesion (total fluctuation divided by lesion duration) was calculated and expressed in $\mathrm{mg} / \mathrm{s}$. Variation was greatest Mid CTI (table 3) and was amplified in lesions with higher peak AI values. However, use of a long sheath significantly reduced temporal fluctuations in contact force throughout the CTI (table 3, figure 3).

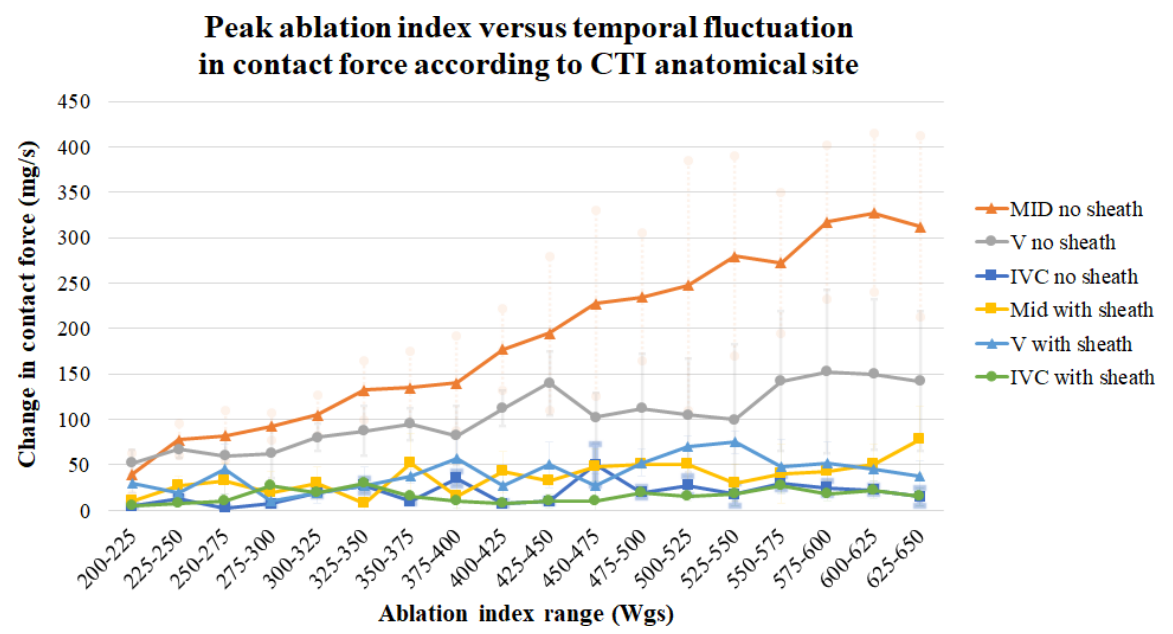

Figure 3: Average temporal fluctuation in contact force $(\mathrm{mg} / \mathrm{s})$ per ablation lesion according to peak ablation index, stratified according to CTI anatomical site and the use of a long sheath.

Table 3: Variation in contact force across the CTI

\begin{tabular}{|c|c|c|c|c|c|}
\hline Parameter & Whole CTI & IVC & Middle & Ventricular & p value \\
\hline $\begin{array}{l}\text { Temporal } \\
\text { fluctuation } \\
\text { in contact } \\
\text { force }(\mathrm{mg} / \mathrm{s}) \\
\text { - no sheath } \\
\text { used }\end{array}$ & $103.5 \pm 93.8$ & $19.3 \pm 12.0$ & $188.8 \pm 92.1$ & $102.8 \pm 32.3$ & All $<0.0001$ \\
\hline $\begin{array}{l}\text { Temporal } \\
\text { fluctuation in } \\
\text { contact force } \\
(\mathrm{mg} / \mathrm{s})-\text { long } \\
\text { sheath used }\end{array}$ & $31.0 \pm 18.3$ & $16.1 \pm 7.3$ & $36.5 \pm 17.2$ & $40.8 \pm 17.7$ & $\begin{array}{l}\text { IVC vs Mid or } \\
\text { IVC vs V } \\
<0.0001, \text { Mid vs } \\
\text { V p }=0.085\end{array}$ \\
\hline p value & $<0.0001$ & 0.025 & $<0.0001$ & $<0.0001$ & \\
\hline
\end{tabular}

Mean temporal fluctuation in contact force $(\mathrm{mg} / \mathrm{s})$ per ablation lesion stratified according to CTI anatomical site and the use of a long sheath. Significant p values $(<0.05)$ are highlighted in bold.

Fluctuation in catheter angle of incidence was assessed in both the axial and lateral planes. For each lesion, 
change in catheter angle was recorded every $10-20 \mathrm{~ms}$ and the average value determined in degrees/s. Variations in catheter tip angle were most marked at the Mid CTI (Lateral plane - IVC: $0.82 \pm 0.4$ degrees/s, Mid: $2.83 \pm 1.1$ degrees/s, V: $1.0 \pm 0.6$ degrees/s, p $<0.0001 ;$ Axial plane- IVC: $0.42 \pm 0.2$ degrees/s, Mid: $3.4 \pm 1.3$ degrees/s, V: $0.28 \pm 0.2$ degrees/s, $\mathrm{p}<0.0001$ ) but again diminished with the use of a long sheath (figure 4 ).

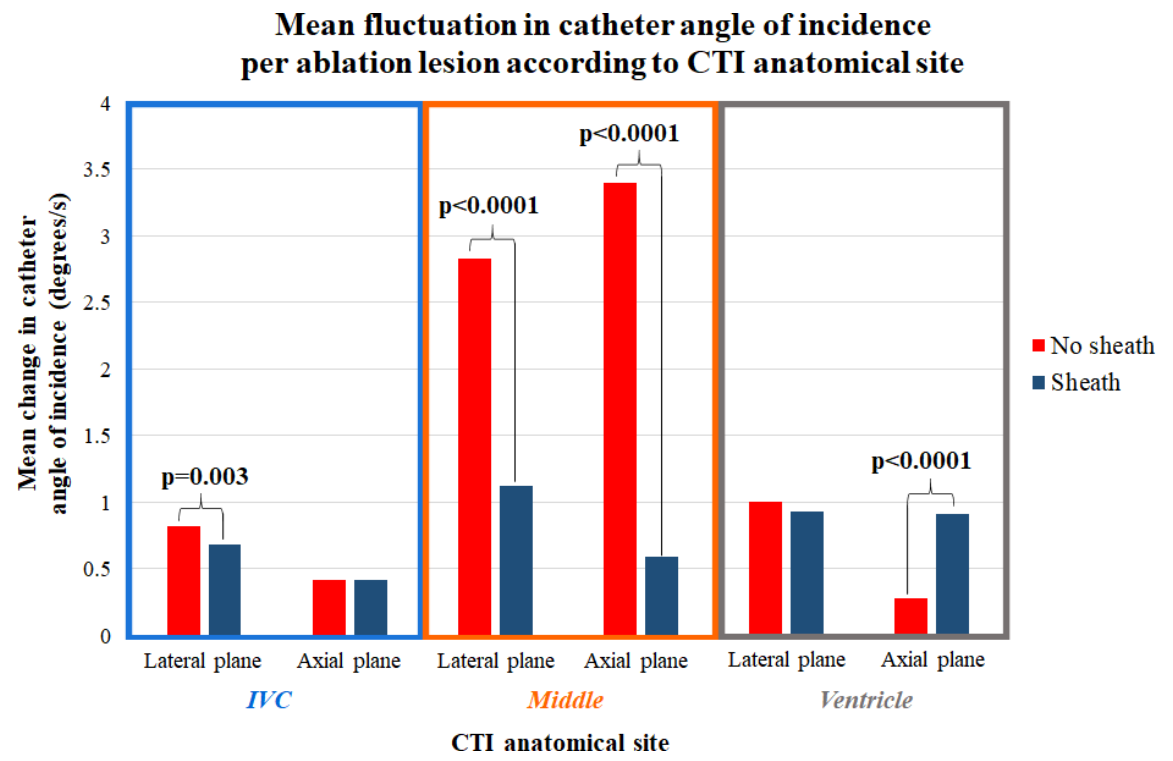

Figure 4: Average temporal fluctuation in catheter angle of incidence (degrees/s) in the lateral and axial planes for each ablation lesion, stratified according to CTI anatomical site and the use of a long sheath. Significant p values highlighted; other relationships non-significant.

As these findings inferred that the use of a long sheath attenuated the catheter tip instability seen at the Mid CTI, the relationship between AI and ID was re-examined according to sheath use (table 4).

Table 4: Ablation lesion characteristics at the Mid CTI according to sheath use

\begin{tabular}{lll}
\hline Parameter & Middle CTI: no sheath $(\mathbf{n = 1 5 2})$ & Middle CTI: long sheath used $(\mathbf{n = 9 0}$ \\
\hline Peak AI (Wgs) & $549.2 \pm 103.5$ & $523.4 \pm 99.6$ \\
Power (W) & $45.0 \pm 4.9$ & $44.5 \pm 4.1$ \\
Lesion duration (s) & $30.9 \pm 13.2$ & $25.5 \pm 14.3$ \\
Contact force (g) & $10.2 \pm 6.6$ & $11.2 \pm 8.1$ \\
Impedance drop (Ohms) & $8.2 \pm 7.4$ & $10.4 \pm 7.1$ \\
Rate of impedance drop (Ohms/s) & $0.15 \pm 0.12$ & $0.23 \pm 0.16$ \\
\hline
\end{tabular}

Significant $\mathrm{p}$ values $(<0.05)$ are highlighted in bold.

Despite a trend towards lower peak AI, use of a long sheath at the Mid CTI was associated with a greater and more rapid drop in impedance.

Discussion

Our study made use of Ablation Index (AI) to examine ablation lesion characteristics in the treatment of typical atrial flutter. We found that using a target AI of 600Wgs across the CTI was safe, but there were significant inconsistencies in energy delivery according to anatomical site, with particularly notable variance seen in the Mid CTI. 
Whilst mean contact force and power were similar across all CTI sites, higher peak AI values were seen Mid CTI, which appears to be mediated through compensatory increases in lesion duration. However, at this site, increased peak AI values did not necessarily correlate with greater impedance drop, and both the rate of impedance drop and the mean lesion temperature were significantly lower at this site despite similar catheter tip power, mean contact force and irrigation techniques, suggestive of inferior lesion efficacy.

We propose this occurs because of significant temporal fluctuations in contact force and catheter tip angle which are most exaggerated at the Mid CTI (figures $3 \& 4$ ). It is accepted that changes in catheter angle of incidence affect lesion size, and that consistent tissue contact produces larger lesions than intermittent contact (20). The fluctuations seen in our study are likely to indicate catheter tip instability encountered due to established anatomical anomalies Mid CTI (13). Consequently, whilst the relationship between AI and ID is mostly linear (figures $1 \& 2$ ) - and hence peak AI is a reasonable surrogate for lesion efficacy in atrial flutter - we suggest that operators should exercise caution at the Mid CTI, where higher peak AI values do not necessarily equate to more effective ablation.

The finding that fluctuations in contact force Mid CTI become more pronounced at higher AI values is unexpected (figure 3); we suggest this may relate to the accumulation of local tissue oedema as a result of more prolonged radiofrequency applications, which may in turn further retard impedance drop - this hypothesis could not be assessed in our study. Importantly, the use of a long sheath appears to confer additional stability to the catheter tip and overcome the majority of inconsistencies in force and angle, although a significant increase in axial plane fluctuation was noted at the ventricular margin (figure 4). This finding may represent catheter and sheath overreach at the point of the valve annulus into the ventricular inflow tract; a long sheath remained effective in stabilising contact force at this site.

The optimal AI for safe and effective CTI ablation is not well established; our target AI of 600Wgs was based on retrospective analysis performed at our centre (18), and is in excess of that which is generally recommended for left atrial procedures. Importantly, we did not record any acute or long-term complications with our protocol for CTI ablation. Our mechanistic study was not designed to assess long-term procedural efficacy, however it is encouraging that $97.4 \%$ of patients were in sinus rhythm after 6 months' follow-up. Longer term clinical review is in progress.

To our knowledge, we are the second group to analyse AI in the ablation of atrial flutter. Zhang et al. (2019) compared AI-guided ablation versus contact-force guided ablation of the CTI, and found higher rates of first-pass conduction block in their AI-guided group (21). These authors delivered ablation with AI targets of 500Wgs to the anterior two thirds of the CTI and 400Wgs to the posterior third; these target values were derived from studies of PVI and adjusted according to accepted variations in CTI thickness. They found that acute reconnection of the CTI was more common at the ventricular aspect of the CTI with AI values of $<450 \mathrm{Wgs}$. In contrast to our study, the authors' protocol delivered higher AI values at the anterior segments and found that this was associated with a relatively larger drop in impedance. Temporal changes in contact force or catheter angle, and the correlation of impedance drop with different peak AI values, were not examined. Our study is the first to make use of precise AI-associated lesion delivery characteristics to define the heterogeneity encountered when ablating the CTI.

Whilst our findings may have been more sharply delineated with the addition of peri- or -intraprocedural imaging studies, in clinical practice outcomes for CTI ablation without these techniques are already reasonable . Accordingly, our aim was to explore a novel utility of an existing technology as a vehicle for incremental improvements in procedural success and safety, which could be adopted clinically without considerable additional resources. We have examined the correlation of pre-specified AI targets with post hoc values of mean ID at the CTI; a multi-centre trial (LOCALIZE) assessing the efficacy of PVI guided by live measurements of local impedance is ongoing, and may further contribute to our understanding of predicting lesion transmurality from intraprocedural variables (22).

Our study has important limitations. Whilst our patient sample is multicentre, patient numbers are small; the number of extracted lesion data points was sufficient to permit key analyses, however meaningful comparisons 
of additional potential confounders - for example catheter curve, patient co-morbidities, or the use of general anaesthetic or of class III anti-arrhythmic drugs - could not be performed. AI itself is validated only for ablation catheters made by Biosense Webster; our results may not be generalisable to equipment from other manufacturers. Likewise, our operators did not use surround flow (STSF) catheters, which are known to have different biophysical efficacy than their ST equivalent and hence our findings cannot be extrapolated to this technology. We used a measure of impedance drop as marker of ablation efficacy; this is a widely accepted surrogate in the literature however histological analysis is the gold standard for the assessment of lesion quality and this was not available in our study.

\section{Conclusions}

In the ablation of CTI-dependent atrial flutter, the use of ablation index (AI) appears safe when delivering lesions up to 600Wgs. Whilst AI is generally a reliable marker of ablation efficacy by impedance drop, we suggest that operators exercise caution when interpreting AI values at the Mid CTI, where anatomical anomalies may introduce catheter instability and hence impede energy delivery. Higher AI values are required Mid CTI to deliver lesions comparable to the CTI margins, and this phenomenon seems to be mediated by temporal fluctuations in contact force and catheter angle. It appears that the use of a long sheath may mitigate these fluctuations and restore a more linear relationship between AI and ID. In the absence of a long sheath, an ablation strategy which prescribes higher AI targets to the Mid CTI may improve acute lesion efficacy and hence long-term outcomes, however this requires prospective validation.

\section{REFERENCES}

1. Thiagalingam A, D'Avila A, Foley L, Guerrero JL, Lambert H, Leo G, Ruskin JN, Reddy VY Importance of Catheter Contact Force During Irrigated Radiofrequency Ablation: Evaluation in a Porcine Ex Vivo Model Using a Force-Sensing Catheter Journal of Cardiovascular Electrophysiology, 2010, 21 (7)

2. Yokoyama K, Nakagawa H, Shah DC, Lambert H, Leo G, Aeby N, Ikeda A, Pitha JV, Sharma T, Lazzara R, Jackman WM Contact Force Sensor Incorporated in Irrigated Radiofrequency Ablation Catheter Predicts Lesion Size and Incidence of Steam Pop and Thrombus. Circulation: Arrhythmia \& Electrophysiology, 2008, Vol. 1 (5).

3. Reddy V, Shah D, Kautzner J, Schmidt B, Saoudi N, Herrera C, Jais P, Hindricks G, Peichl P, Yulzari A, Lambert H, Neuzil P, Natale A, Kuck K-H The relationship between contact force and clinical outcome during radiofrequency catheter ablation of atrial fibrillation in the TOCCATA study. Heart Rhythm, 2012, Vol. 9 (11).

4. Nakagawa H, Ikeda A, Govari A, Papaioannou T, Constantine G, Bar-Tal M. Prospective study to test the ability to create RF lesions at predicted depths of 3, 5, 7 and $9 \mathrm{~mm}$ using a new formula incorporating contact force, radiofrequency power and application time (Force-Power-Time Index) in the beating canine heart. Heart Rhythm, 2013, vol. 10., S481

5. Hussein A, Das M, Chaturvedi V, Asfour IK, Daryanani N, Morgan M, Ronayne C, Shaw M, Snowdon $\mathrm{R}$, Gupta D Prospective use of Ablation Index targets improves clinical outcomes following ablation for atrial fibrillation. Journal of Cardiovascular Electrophysiology, 2017, Vol. 28. (9)

6. Pranata R, Vania R, Huang I Ablation-index guided versus conventional contact-force guided ablation in pulmonary vein isolation - Systematic review and meta-analysis. Indian Pacing Electrophysiol J., 2019, Vol. 19. (4) 7. Santoro F, Metzner A, Brunetti ND, Heeger C-H, Mathew S, Reissman B, Lemes C, Maurer T, Fink T, Rottner L, Inaba O, Kuck K-H, Ouyang F, Rillig A Left atrial anterior line ablation using ablation index and inter-lesion distance measurement. Clinical Research in Cardiology, 2019, Vol. 108. (9) 8. Phlips T, Taghji P, El Haddad M, Wolf M, Knecht S, Vandekerckhove Y, Tavernier R, Duytschaever M. Improving procedural and one-year outcome after contact force-guided pulmonary vein isolation: the role of interlesion distance, ablation index, and contact force variability in the 'CLOSE'-protocol. Europace, 2018, Vol. 1. 9. Ullah W, Hunter RJ, Finlay MC, McLean A, Dhinoja MB, Sporton S, Earley MJ, Schilling RJ Ablation Index and Surround Flow Catheter Irrigation: Impedance-Based Appraisal in Clinical Ablation. JACC: Clinical Electrophysiology, 2017, Vol. 3. (10) 10. Kawaji T, Hojo S, Kushiyama A, Nakatsuma K, Kaneda K, Kato 
M, Yokomatsu T, Miki S Limitations of lesion quality estimated by ablation index: An in vitro study Journal of Cardiovascular Electrophysiology, 2019, Vol. 30. (6) 11. Pérez FJ, Schubert CM, Parvez B, Pathak V, Ellenbogen K, Wood MALong-term outcomes after catheter ablation of cavo-tricuspid isthmus dependent atrial flutter: a meta-analysis. Circ Arrhythm Electrophysiol., 2009, Vol. 2. (4) 12. Brugada J, Katritsis DG, Arbelo E, Arribas F, Bax JJ, Blomström-Lundqvist C, Calkins H, Corrado D, Deftereos SG, Diller GP, Gomez-Doblas JJ, Gorenek B, Grace A, Ho SY, Kaski JC, Kuck KH, Lambiase PD, Sacher F, SarquellaBrugada G, Suwalski P, Zaza A; ESC Scientific Document Group 2019 ESC Guidelines for the management of patients with supraventricular tachycardia. Eur Heart J, 2019, Vol. ehz467. 13. Klimek-Piotrowska W, Hołda MK, Koziej M, Holda J, Piatek K, Tyrak K, Bolechala F Clinical Anatomy of the Cavotricuspid Isthmus and Terminal Crest. PLoS ONE, 2016, Vol. 11. (9) 14. García-Dora J, Pérez-Rodon J, Rodriguez-García J, Sarrias-Merce A, Rivas-Gandara N, Roca-Luque I, Francisco-Pascual J, Santos-Ortega A, Martin-Sanchez G, Ferreira-Gonzalez I, Rodriguez-Palomares J, Evangelista-Masip A, Garcia-Dorado D, Moya-Mitjans A Predictors of acute inefficacy and the radiofrequency energy time required for cavotricuspid isthmus-dependent atrial flutter ablation. Journal of Interventional Cardiac Electrophysiology, 2017, Vol. 49. (1) 15. Paetsch I, Sommer P, Jahnke C, Hilbert S, Loebe S, Schoene K, Oebel S, Krueger S, Weiss S, Smink J, Lloyd T, Hindricks G Clinical workflow and applicability of electrophysiological cardiovascular magnetic resonanceguided radiofrequency ablation of isthmus-dependent atrial flutter: European Heart Journal - Cardiovascular Imaging, 2019, Vol. 20. (2) 16. Kumar S, Morton JB, Lee G, Halloran K, Kistler PM, Kalman JM.High Incidence of Low Catheter-Tissue Contact Force at the Cavotricuspid Isthmus During Catheter Ablation of Atrial Flutter: Implications for Achieving Isthmus Block. J Cardiovasc Electrophysiol., 2015, Vol. 26. (8) 17. Baccillieri MS, Rizzo S, De Gaspari M, Paradiso B, Thiene G, Verlato R, Basso C Anatomy of the cavotricuspid isthmus for radiofrequency ablation in typical atrial flutter. Heart Rhythm, 2019, Vol. 16. (11) 18. Maclean E, Simon R, Dhillon G, Ahsan S, Hunter RJ, Behar, JMUse of ablation index to understand lesion delivery in the era of contact force guided ablation for cavo-tricuspid isthmus dependent atrial flutter. European Journal of Arrhythmia and Electrophysiology, 2019, Vol. 5. (Suppl. 1) 19. Reichlin T, Lane C, Nagashima K, Nof E, Chopra N, Ng J, Barbhaiya C, Tadros T, John R, Stevenson WG, Michaud GF Feasibility, efficacy and safety of radiofrequency ablation of atrial fibrillation guided by monitoring of the initial impedance decrease as a surrogate of catheter contact J Cardiovasc Electrophysiol. 2015, 26 (4), 390396 20. Shah D, Lambert H, Nakagawa H, Langenkamp A, Aeby N, Leo G.Area Under the Real-Time Contact Force Curve (Force-Time Integral) Predicts Radiofrequency Lesion Size in an in Vitro Contractile Model J Cardiovasc Electrophysiol., 2010, 21 (9) 21. Zhang T, Wang Y, Han Z, Zhao H, Liang Z, Wang Y, Wu Y, Ren $\mathrm{X}$ Cavotricuspid isthmus ablation using ablation index in typical right atrial flutter. Journal of Cardiovascular Electrophysiology, 2019, Vol. 30. (11) 22. Retrieved from https://clinicaltrials.gov/ct2/show/NCT03232645: Electrical Coupling Information From The Rhythmia HDx System And DirectSense Technology In Subjects With Paroxysmal Atrial Fibrillation (LOCALIZE)

\section{FIGURE LEGENDS}

Figure 1: Relationship between peak ablation index (Wgs) and mean impedance drop (Ohms; mean \pm SD) for all CTI lesions. Pearson's $\mathrm{R}^{2}=0.89, \mathrm{p}<0.0001$.

Figure 2: Relationship between peak ablation index (Wgs) and mean impedance drop (Ohms) per lesion according to CTI anatomical site (V CTI $\mathrm{R}^{2}=0.95, \mathrm{p}<0.0001$, Mid CTI $\mathrm{R}^{2}=0.15, \mathrm{p}=0.21$, IVC CTI $\mathrm{R}^{2}=0.88$, $\mathrm{p}<0.0001)$.

Figure 3: Average temporal fluctuation in contact force $(\mathrm{mg} / \mathrm{s})$ per ablation lesion according to peak ablation index, stratified according to CTI anatomical site and the use of a long sheath.

Figure 4: Average temporal fluctuation in catheter angle of incidence (degrees/s) in the lateral and axial planes for each ablation lesion, stratified according to CTI anatomical site and the use of a long sheath. Significant $\mathrm{p}$ values highlighted; other relationships non-significant. 